\title{
Analysis of mean function discrete LSM-estimator for biperiodically nonstationary random signals
}

\author{
Javorskyj I. ${ }^{1,2}$, Dzeryn O. ${ }^{1}$, Yuzefovych R..$^{1,3}$ \\ ${ }^{1}$ Karpenko Physico-Mechanical Institute of National Academy of Sciences of Ukraine, \\ Laboratory of vibration-based diagnosis, 5 Naukova Str., 79060, Lviv, Ukraine \\ ${ }^{2}$ UTP University of Sciences and Technology, \\ Institute of Telecommunication and Computer Science, \\ Al. Prof. S. Kaliskiego 7, 85796, Bydgoszcz, Poland \\ ${ }^{3}$ Lviv Polytechnic National University, Department of Applied Mathematics, \\ 12 Bandera Str., 79013, Lviv, Ukraine
}

(Received 3 December 2018; Revised 29 March 2019; Accepted 30 March 2019)

\begin{abstract}
Discrete estimators of the deterministic part for a biperiodically nonstationary signal obtained by the least square method (LSM) are analysed. It was shown that LSM-estimation allows avoiding the leakage effects. The conditions of consistency for the discrete estimators are obtained. The formulae for variance estimators, which describe their dependencies on a realization length, sampling interval and signal covariance components, are analysed.

Keywords: biperiodically correlated random process, the least square method, discrete estimators of deterministic part parameters, unbiasedness of estimate, consistency.
\end{abstract}

2000 MSC: 60G35, 94A12

UDC: $621.391: 519.72$

DOI: $10.23939 / \mathrm{mmc} 2019.01 .044$

\section{Introduction}

While researching time variability regularities of both natural and man-made physical processes, the situation often occurs, when the single stochastic periodicity is overlapped with other periodicity. For example, the diurnal and annual cycles, including so-called eleven-year periodic change in the Sun's activity, are the specific features of time variations for many geophysical processes [1]. The diurnal, weekly, and annual cycles are manifested in the changes of the electric power consumption [2]. The biperiodic recurrence also appears in the properties of communication signals [3]. These recurrences are caused both by periodical changes of the carrier and rhythmic variability of information signals. In vibration signals, polyrhythmic nature is caused primarily by the different rotation frequencies of mechanical system units $[4,5]$. The binary stochastic recurrence can be adequately described by biperiodically correlated random processes (BPCRP) [6-8].

The BPCRP mean function $m(t)=E \xi(t)$ and its covariance function $b(t, u)=E \stackrel{\circ}{\xi}(t) \stackrel{\circ}{\xi}(t+u)$, $\stackrel{\circ}{\xi}(t)=\xi(t)-m(t)$, where $E$ is the probability distribution mean operator, are determined by the following formulae:

$$
\begin{aligned}
m(t) & =\sum_{l, k \in \mathbb{Z}} m_{k l} e^{i \Lambda_{k l} t}, \quad \Lambda_{k l}=k \frac{2 \pi}{T_{1}}+l \frac{2 \pi}{T_{2}}, \\
b(t, u) & =\sum_{l, k \in \mathbb{Z}} B_{k l}(u) e^{i \Lambda_{k l} t}
\end{aligned}
$$

where $T_{1}$ and $T_{2}$ are positive quantities called the periods of binonstationarity. Hereafter, we accept the number of harmonics with basic frequencies $\Lambda_{k 0}$ and $\Lambda_{0 l}$ and also with combinative frequencies $\Lambda_{k l}$ is finite and $k=\overline{-N_{1}, N_{1}}, l=\overline{-N_{1}, N_{1}}$ for mean function, $k=\overline{-N_{2}, N_{2}}$ and $l=\overline{-N_{2}, N_{2}}$ for covariance 
function. It is obvious that $\Lambda_{-k,-l}=-\Lambda_{k l}$ and $m_{-k,-l}=\overline{m_{k, l}}$, and also $B_{-k,-l}(u)=\overline{B_{k, l}(u)}$, where "-" is a conjugation sign.

Fourier coefficients of time-series (1) and (2) are theoretically determined by the following limit ratios:

$$
\begin{aligned}
m_{k l} & =\lim _{\theta \rightarrow \infty} \frac{1}{2 \theta} \int_{-\theta}^{\theta} m(t) e^{-i \Lambda_{k l}} d t, \\
B_{k l}(u) & =\lim _{\theta \rightarrow \infty} \frac{1}{2 \theta} \int_{-\theta}^{\theta} b(t, u) e^{-i \Lambda_{k l}} d t .
\end{aligned}
$$

While processing experimental data, we deal with realizations of the finite length. Statistics for coefficients $m_{k l}$ estimation built analogically to (3) in discrete form are the following [8]:

$$
\hat{m}_{k l}=\frac{1}{K} \sum_{n=0}^{K-1} \xi(n h) e^{-i \Lambda_{k l} n h}
$$

where $h=\frac{\theta}{K}$ is the sampling interval, $K \in \mathbb{N}$. The mathematical expectation of estimator (4) is equal to

$$
E \hat{m}_{k l}=\sum_{p, q=-N_{1}}^{N_{1}} m_{p q} \varphi\left(\Lambda_{p-k, q-l} K\right),
$$

where $\varphi\left(\Lambda_{p-k, q-l} K\right)=K^{-1} \sum_{n=0}^{K-1} e^{i \Lambda_{p-k, q-l} n h}$. Then

$$
\hat{m}(n h)=m(n h)+\sum_{k, l=-N_{1}}^{N_{1}} \varepsilon\left[\hat{m}_{k l}\right] e^{i \Lambda_{k l} n h} .
$$

The values

$$
\varepsilon\left[\hat{m}_{k l}\right]=\sum_{\substack{p, q=-N_{1} \\ p \neq k, q \neq l}}^{N_{1}} m_{p q} \varphi\left(\Lambda_{p-k, q-l} K\right)
$$

define the systematic errors of the component discrete estimation. The errors caused by aliasing effects are avoided if sampling interval $h$ satisfies the inequality $h<\pi / \Lambda_{N_{1} N_{1}}$. The leakage errors decrease to zero if $K \rightarrow \infty$, and, for finite $K$, they are absent only in the cases when $\theta=P T_{1}=M T_{2}$, where $P$ and $M$ are natural numbers. This condition is difficult to implement in practice. Using the least square method (LSM), to estimate BPCRP mean function, we obtain unbiased estimators for arbitrary $\theta$ and frequencies of harmonics of series (1). Mean-square error of LSM-estimator is determined by the mean-square convergence that depends on the number $K$ and sampling interval $h$. The grounded choice of these statistical processing parameters can be made based on discrete LSM-estimators analysis.

\section{The properties of LSM-estimators}

Let us consider the estimation method based on minimization of the functional

$$
F\left(\hat{m}_{0}, \hat{m}_{11}^{c}, \ldots, \hat{m}_{k l}^{c}, \ldots, \hat{m}_{N_{1} N_{1}}^{s}\right)=\sum_{n=0}^{K-1}[\xi(n h)-\hat{m}(n h)]^{2},
$$

where

$$
\hat{m}_{k l}=\frac{1}{2}\left[\hat{m}_{k l}^{c}-i \hat{m}_{k l}^{s}\right] .
$$

Mathematical Modeling and Computing, Vol.6, No. 1, pp. 44-57 (2019) 
To make the following analysis, let us represent the BPCRP mean function and its estimator in a real form. Taking into account $m_{-k,-l}=\overline{m_{k l}}$, we obtain

$$
\begin{aligned}
& m(n h)=\sum_{k=-N_{1}}^{-1}\left[\sum_{l=-N_{1}}^{-1} m_{k l} e^{i \Lambda_{k l} n h}+\sum_{l=0}^{N_{1}} m_{k l} e^{i \Lambda_{k l} n h}\right]+\sum_{k=0}^{N_{1}}\left[\sum_{l=-N_{1}}^{-1} m_{k l} e^{i \Lambda_{k l} n h}+\sum_{l=0}^{N_{1}} m_{k l} e^{i \Lambda_{k l} n h}\right] \\
= & m_{00}+\sum_{k=0}^{N_{1}} \sum_{l=1}^{N_{1}}\left(m_{k l}^{c} \cos \left(\Lambda_{k l} n h\right)+m_{k l}^{s} \sin \left(\Lambda_{k l} n h\right)\right)+\sum_{l=0}^{N_{1}} \sum_{k=1}^{N_{1}}\left(m_{k,-l}^{c} \cos \left(\Lambda_{k,-l} n h\right)+m_{k,-l}^{s} \sin \left(\Lambda_{k,-l} n h\right)\right) .
\end{aligned}
$$

Then

$$
\begin{aligned}
F\left(\hat{m}_{00}, \hat{m}_{11}, \ldots, \hat{m}_{k l}^{c}, \ldots, \hat{m}_{N_{1} N_{1}}^{s}\right)=\sum_{n=0}^{K-1}[ & \xi(n h)-\left[\hat{m}_{00}+\sum_{k=0}^{N_{1}} \sum_{l=1}^{N_{1}}\left(\hat{m}_{k l}^{c} \cos \left(\Lambda_{k l} n h\right)+\hat{m}_{k l}^{s} \sin \left(\Lambda_{k l} n h\right)\right)\right. \\
& \left.\left.+\sum_{l=0}^{N_{1}} \sum_{k=1}^{N_{1}}\left(\hat{m}_{k,-l}^{c} \cos \left(\Lambda_{k,-l} n h\right)+\hat{m}_{k,-l}^{s} \sin \left(\Lambda_{k,-l} n h\right)\right)\right]\right]^{2} .
\end{aligned}
$$

The estimators for quantities $\hat{m}_{00}, \hat{m}_{k l}^{c}, \hat{m}_{k l}^{s}, \hat{m}_{k,-l}^{c}$, and $\hat{m}_{k,-l}^{s}$ can be found by solving the linear equations system that provide necessary conditions for the functional (5) minimum existence

$$
\left.\left.\frac{\partial F}{\partial \hat{m}_{00}}=0, \quad \begin{array}{c}
\frac{\partial F}{\partial \hat{m}_{k l}^{c}}=0 \\
\frac{\partial F}{\partial \hat{m}_{k l}^{s}}=0
\end{array}\right\} \begin{array}{cc}
k=\overline{0, N_{1}}, & \frac{\partial F}{\partial \hat{m}_{k,-l}^{c}}=0 \\
l=\overline{1, N_{1}}, & \frac{\partial F}{\partial \hat{m}_{k,-l}^{s}}=0
\end{array}\right\} \begin{gathered}
k=\overline{1, N_{1}}, \\
l=\overline{0, N_{1}} .
\end{gathered}
$$

This is a linear equations system:

$$
\begin{gathered}
K \hat{m}_{00}+\sum_{k=0}^{N_{1}} \sum_{l=1}^{N_{1}}\left[\hat{m}_{k l}^{c} \sum_{n=0}^{K-1} \cos \left(\Lambda_{k l} n h\right)+\hat{m}_{k l}^{s} \sum_{n=0}^{K-1} \sin \left(\Lambda_{k l} n h\right)\right] \\
+\sum_{l=0}^{N_{1}} \sum_{k=1}^{N_{1}}\left[\hat{m}_{k,-l}^{c} \sum_{n=0}^{K-1} \cos \left(\Lambda_{k,-l} n h\right)+\hat{m}_{k,-l}^{s} \sum_{n=0}^{K-1} \sin \left(\Lambda_{k l} n h\right)\right]=\sum_{n=0}^{K-1} \xi(n h), \\
\hat{m}_{00} \sum_{n=0}^{K-1} \cos \left(\Lambda_{r s} n h\right)+\sum_{k=0}^{N_{1}} \sum_{l=1}^{N_{1}} \hat{m}_{k l}^{c} \sum_{n=0}^{K-1} \cos \left(\Lambda_{k l} n h\right) \cos \left(\Lambda_{r s} n h\right)+\hat{m}_{k l}^{s} \sum_{n=0}^{K-1} \sin \left(\Lambda_{k l} n h\right) \cos \left(\Lambda_{r s} n h\right) \\
+\sum_{l=0}^{N_{1}} \sum_{k=1}^{N_{1}}\left[\hat{m}_{k,-l}^{c} \sum_{n=0}^{K-1} \cos \left(\Lambda_{k,-l} n h\right) \cos \left(\Lambda_{r s} n h\right)+\hat{m}_{k,-l}^{s} \sum_{n=0}^{K-1} \sin \left(\Lambda_{k l} n h\right) \cos \left(\Lambda_{r s} n h\right)\right] \\
\hat{m}_{00} \sum_{n=0}^{K-1} \sin \left(\Lambda_{r s} n h\right)+\sum_{k=0}^{K-1} \sum_{l=1}^{N_{1}} \hat{m}_{k l}^{s} \sum_{n=0}^{K-1} \cos \left(\Lambda_{k l} n h\right) \sin \left(\Lambda_{r s} n h\right)+\hat{m}_{k,-l}^{s} \sum_{n=0}^{K-1} \sin \left(\Lambda_{k l} n h\right) \sin \left(\Lambda_{r s} n h\right) \\
+\sum_{l=0}^{N_{1}} \sum_{k=1}^{N_{1}}\left[\hat{m}_{k,-l}^{c} \sum_{n=0}^{K-1} \cos \left(\Lambda_{k,-l} n h\right) \sin \left(\Lambda_{r s} n h\right)+\hat{m}_{k,-l}^{s} \sum_{n=0}^{K-1} \sin \left(\Lambda_{k l} n h\right) \sin \left(\Lambda_{r s} n h\right)\right] \\
n_{n=0}^{K-1} \xi(n h) \sin \left(\Lambda_{r s} n h\right)
\end{gathered}
$$

Mathematical Modeling and Computing, Vol. 6, No. 1, pp. 44-57 (2019) 
For the last two equations $r, s=\overline{-N_{1}, N_{1}}, r=s \neq 0$. To simplify the following analysis of the solutions of system (6), it is expedient to rename harmonic frequencies as well as theirs amplitudes. Table 1 shows harmonic frequencies that produce the mean estimator, and Table 2 shows their respective renaming frequencies.

Table 1. Harmonic frequencies of estimator $\hat{m}(n h)$.

\begin{tabular}{|c|c|c|c|c|c|c|c|c|}
\hline$\Lambda_{N_{1},-N_{1}}$ & $\Lambda_{N_{1},-N_{1}+1}$ & $\ldots$ & $\Lambda_{N_{1},-1}$ & $\Lambda_{N_{1} 0}$ & $\Lambda_{N_{1} 1}$ & $\Lambda_{N_{1} 2}$ & $\ldots$ & $\Lambda_{N_{1} N_{1}}$ \\
\hline$\Lambda_{N_{1}-1,-N_{1}}$ & $\Lambda_{N_{1}-1,-N_{1}+1}$ & $\ldots$ & $\Lambda_{N_{1}-1,-1}$ & $\Lambda_{N_{1}-1,0}$ & $\Lambda_{N_{1}-1,1}$ & $\Lambda_{N_{1}-1,2}$ & $\ldots$ & $\Lambda_{N_{1}-1, N_{1}}$ \\
\hline$\ldots$ & $\ldots$ & $\ldots$ & $\ldots$ & $\ldots$ & $\ldots$ & $\ldots$ & $\ldots$ & $\ldots$ \\
\hline$\Lambda_{2,-N_{1}}$ & $\Lambda_{2,-N_{1}+1}$ & $\ldots$ & $\Lambda_{2,-1}$ & $\Lambda_{2,0}$ & $\Lambda_{2,1}$ & $\Lambda_{2,2}$ & $\ldots$ & $\Lambda_{2, N_{1}}$ \\
\hline$\Lambda_{1,-N_{1}}$ & $\Lambda_{1,-N_{1}+1}$ & $\ldots$ & $\Lambda_{1,-1}$ & $\Lambda_{1,0}$ & $\Lambda_{1,1}$ & $\Lambda_{1,2}$ & $\ldots$ & $\Lambda_{1, N_{1}}$ \\
\hline & & & & & $\Lambda_{0,1}$ & $\Lambda_{0,2}$ & $\ldots$ & $\Lambda_{0, N_{1}}$ \\
\hline
\end{tabular}

Table 2. Renaming frequencies of estimator $\hat{m}(n h)$.

\begin{tabular}{|c|c|c|c|c|c|c|c|c|}
\hline$\omega_{2 N_{1}\left(N_{1}+1\right)}$ & $\cdots$ & $\omega_{N_{1}\left(2 N_{1}+1\right)}$ & $\omega_{N_{1}\left(2 N_{1}+1\right)}$ & $\omega_{N_{1}^{2}+1}$ & $\omega_{N_{1}^{2}+2}$ & $\cdots$ & $\omega_{N_{1}^{2}+N_{1}-1}$ & $\omega_{N_{1}\left(N_{1}+1\right)}$ \\
\hline$\omega_{N_{1}\left(2 N_{1}+1\right)-1}$ & $\cdots$ & $\omega_{2 N_{1}^{2}}$ & $\omega_{2 N_{1}^{2}-1}$ & $\omega_{\left(N_{1}-1\right) N_{1}+1}$ & $\omega_{\left(N_{1}-1\right) N_{1}+2}$ & $\cdots$ & $\omega_{N_{1}^{2}-1}$ & $\omega_{N_{1}^{2}}$ \\
\hline \multicolumn{3}{|c|}{$\cdots$} & $\cdots$ & $\cdots$ & $\cdots$ & $\cdots$ & $\cdots$ & $\cdots$ \\
\hline$\omega_{N_{1}\left(N_{1}+3\right)+2}$ & $\cdots$ & $\omega_{N_{1}\left(N_{1}+2\right)+2}$ & $\omega_{N_{1}\left(N_{1}+2\right)+2}$ & $\omega_{2 N_{1}+1}$ & $\omega_{2 N_{1}+2}$ & $\cdots$ & $\omega_{3 N_{1}-1}$ & $\omega_{3 N_{1}}$ \\
\hline$\omega_{N_{1}\left(N_{1}+2\right)+1}$ & $\cdots$ & $\omega_{N_{1}\left(N_{1}+1\right)+2}$ & $\omega_{N_{1}\left(N_{1}+1\right)+1}$ & $\omega_{N_{1}+1}$ & $\omega_{N_{1}+2}$ & $\cdots$ & $\omega_{2 N_{1}-1}$ & $\omega_{2 N_{1}}$ \\
\hline & & & & $\omega_{1}$ & $\omega_{2}$ & $\cdots$ & $\omega_{N_{1}-1}$ & $\omega_{N_{1}}$ \\
\hline
\end{tabular}

So, for example, $\omega_{N_{1}\left(N_{1}+1\right)}$ matches the combinative frequency $\Lambda_{N_{1} N_{1}}=N_{1}\left(\frac{2 \pi}{T_{1}}+\frac{2 \pi}{T_{2}}\right)$, and complex amplitude of this harmonic $m_{N_{1}\left(N_{1}+1\right)}=\frac{1}{2}\left[m_{N_{1}\left(N_{1}+1\right)}^{c}-i \hat{m}_{N_{1}\left(N_{1}+1\right)}^{s}\right]$. It is the highest frequency harmonic in the spectrum. After renaming, functional (5) is represented by the following formula:

$$
F\left(\hat{m}_{0}, \hat{m}_{1}^{c}, \ldots, \hat{m}_{L}^{c}, \hat{m}_{1}^{s}, \ldots, \hat{m}_{L}^{s}\right)=\sum_{n=0}^{K-1}\left[\xi(n h)-\left(\hat{m}_{0}+\sum_{r=1}^{L}\left(\hat{m}_{r}^{c} \cos \left(\omega_{r} n h\right)+\hat{m}_{r}^{s} \sin \left(\omega_{r} n h\right)\right)\right)\right]^{2},
$$

where $L=2 N_{1}\left(N_{1}+1\right)$. Necessary conditions for the function minimum existence

$$
\frac{\partial F}{\partial \hat{m}_{0}}=0, \quad \frac{\partial F}{\partial \hat{m}_{r}^{c}}=0, \quad \frac{\partial F}{\partial \hat{m}_{r}^{s}}=0, \quad r=\overline{1, L}
$$

now can be rewritten in the following form:

$$
\hat{m}_{0} K+\sum_{k=1}^{L}\left[\hat{m}_{k}^{c} \sum_{n=0}^{K-1} \cos \left(\omega_{k} n h\right)+\hat{m}_{k}^{s} \sum_{n=0}^{K-1} \sin \left(\omega_{k} n h\right)\right]=\sum_{n=0}^{K-1} \xi(n h),
$$

$$
\begin{array}{r}
\hat{m}_{0} \sum_{n=0}^{K-1} \cos \left(\omega_{r} n h\right)+\sum_{k=1}^{L}\left[\hat{m}_{k}^{c} \sum_{n=0}^{K-1} \cos \left(\omega_{k} n h\right) \cos \left(\omega_{r} n h\right)+\hat{m}_{k}^{s} \sum_{n=0}^{K-1} \sin \left(\omega_{k} n h\right) \cos \left(\omega_{r} n h\right)\right] \\
=\sum_{n=0}^{K-1} \xi(n h) \cos \left(\omega_{r} n h\right),
\end{array}
$$

$$
\begin{array}{r}
\hat{m}_{0} \sum_{n=0}^{K-1} \sin \left(\omega_{r} n h\right)+\sum_{k=1}^{L}\left[\hat{m}_{k}^{c} \sum_{n=0}^{K-1} \cos \left(\omega_{k} n h\right) \sin \left(\omega_{r} n h\right)+\hat{m}_{k}^{s} \sum_{n=0}^{K-1} \sin \left(\omega_{k} n h\right) \sin \left(\omega_{r} n h\right)\right] \\
=\sum_{n=0}^{K-1} \xi(n h) \sin \left(\omega_{r} n h\right) .
\end{array}
$$

Mathematical Modeling and Computing, Vol.6, No. 1, pp. 44-57 (2019) 
Let us introduce the following notations:

$$
\begin{gathered}
c_{r k}=\frac{1}{K} \sum_{n=0}^{K-1} \cos \omega_{r} n h \cos \omega_{k} n h, s_{r k}=\frac{1}{K} \sum_{n=0}^{K-1} \sin \omega_{r} n h \sin \omega_{k} n h, a_{k r}=\frac{1}{K} \sum_{n=0}^{K-1} \cos \omega_{r} n h \sin \omega_{k} n h, \\
\tilde{m}_{0}=\frac{1}{K} \sum_{n=0}^{K-1} \xi(n h), \tilde{m}_{r}=\frac{1}{K} \sum_{n=0}^{K-1} \xi(n h) \cos \omega_{r} n h, \tilde{m}_{k}=\frac{1}{K} \sum_{n=0}^{K-1} \xi(n h) \sin \omega_{r} n h .
\end{gathered}
$$

Necessary minimum conditions determining the mean function estimator are the following:

$$
\begin{gathered}
\hat{m}_{0}+\sum_{k=1}^{N_{1}}\left(\hat{m}_{k}^{c} c_{k 0}+\hat{m}_{k}^{s} a_{0 k}\right)=\tilde{m}_{0}, \quad \hat{m}_{0} c_{r 0}+\sum_{k=1}^{N_{1}}\left(\hat{m}_{k}^{c} c_{r k}+\hat{m}_{r}^{s} a_{r k}\right)=\tilde{m}_{r}, \\
\hat{m}_{0} \tilde{a}_{0 r}+\sum_{k=1}^{N_{1}}\left(\hat{m}_{k}^{c} a_{k r}+\hat{m}_{k}^{s} s_{r k}\right)=\tilde{m}_{r+N_{1}}, \quad r=\overline{1, L} .
\end{gathered}
$$

System (7) matrix elements can be easily calculated. We have

$$
\begin{aligned}
c_{r 0} & =\frac{1}{K} \sum_{n=0}^{K-1} \cos \omega_{r} n h=\frac{1}{2 K} \sum_{n=0}^{K-1}\left(e^{i \omega_{r} n h}+e^{-i \omega_{r} n h}\right) \\
& =\frac{\sin K \frac{\omega_{r}}{2} h}{2 K \sin \frac{\omega_{r}}{2} h}\left(e^{i \frac{\omega_{r}}{2}(K-1) h}+e^{-i \frac{\omega_{r}}{2}(K-1) h}\right)=\frac{\sin \frac{\omega_{r}}{2} h \cos (K-1) \frac{\omega_{r}}{2} h}{K \sin \frac{\omega_{r}}{2} h}=S_{1}\left[K, \omega_{r} h\right], \\
a_{0 r} & =\frac{1}{K} \sum_{n=0}^{K-1} \sin \omega_{k} n h=\frac{1}{2 i K} \sum_{n=0}^{K-1}\left(e^{i \omega_{r} n h}-e^{-i \omega_{r} n h}\right) \\
& =\frac{\sin K \frac{\omega_{r}}{2} h}{2 i K \sin \frac{\omega_{r}}{2} h}\left(e^{i \frac{\omega_{r}}{2}(K-1) h}-e^{-i \frac{\omega_{r}}{2}(K-1) h}\right)=\frac{\sin K \frac{\omega_{r}}{2} h \sin \frac{\omega_{r}}{2}(K-1) h}{K \sin \frac{\omega_{r}}{2} h}=S_{2}\left[K, \omega_{r} h\right] .
\end{aligned}
$$

Then,

$$
\begin{aligned}
& c_{r k}=\frac{1}{2 K} \sum_{n=0}^{K-1}\left[\begin{array}{c}
\cos \left(\omega_{k}+\omega_{r}\right) n h \\
+\cos \left(\omega_{k}-\omega_{r}\right) n h
\end{array}\right]=\frac{1}{2}\left[S_{1}\left[K,\left(\omega_{k}+\omega_{r}\right) h\right]+S_{1}\left[K,\left(\omega_{k}-\omega_{r}\right) h\right]\right], \\
& s_{r k}=\frac{1}{2 K} \sum_{n=0}^{K-1}\left[\begin{array}{l}
\cos \left(\omega_{k}+\omega_{r}\right) n h \\
-\cos \left(\omega_{k}+\omega_{r}\right) n h
\end{array}\right]=\frac{1}{2}\left[S_{1}\left[K,\left(\omega_{k}-\omega_{r}\right) h\right]-S_{2}\left[K,\left(\omega_{k}-\omega_{r}\right) h\right]\right], \\
& a_{r k}=\frac{1}{2 K} \sum_{n=0}^{K-1}\left[\begin{array}{l}
\sin \left(\omega_{k}+\omega_{r}\right) n h \\
+\sin \left(\omega_{k}-\omega_{r}\right) n h
\end{array}\right]=\frac{1}{2}\left[S_{2}\left[K,\left(\omega_{k}+\omega_{r}\right) h\right]-S_{2}\left[K,\left(\omega_{k}-\omega_{r}\right) h\right]\right] .
\end{aligned}
$$

The sums are calculated under the condition $h \leqslant \pi / \Lambda_{N_{1} N_{2}}$.

Let us consider matrices

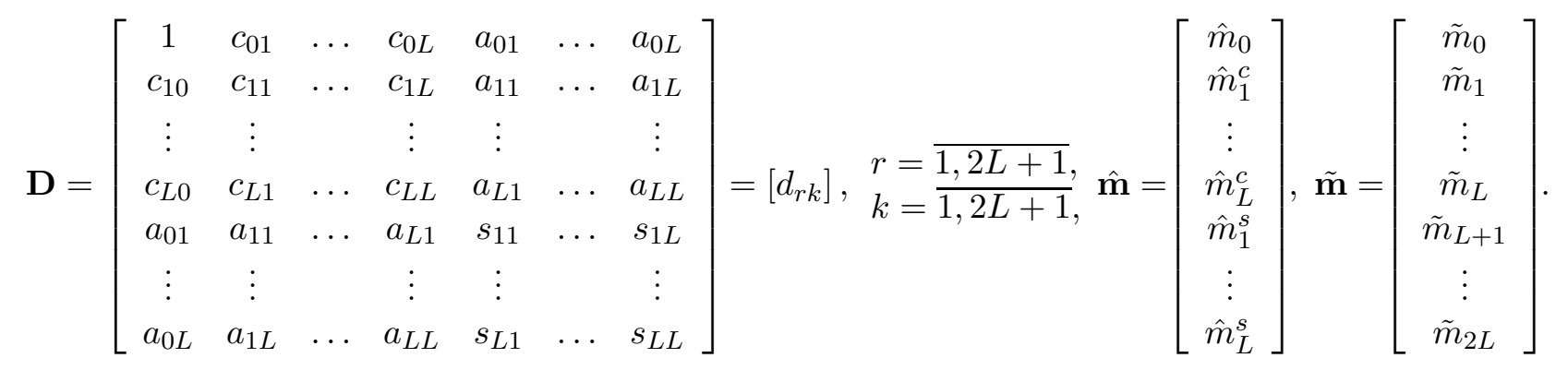


Linear equations system (7) now can be rewritten as a matrix equation:

$$
\mathbf{D} \hat{\mathbf{m}}=\tilde{\mathbf{m}} .
$$

If the rank of matrix $\mathbf{D}$ equals the rank of an expanded matrix $\mathbf{D} \mid \tilde{\mathbf{m}}$, then matrix equality (8) has the only one solution, i.e., the following:

$$
\hat{\mathbf{m}}=\mathbf{D}^{-1} \tilde{\mathbf{m}},
$$

where $\mathbf{D}^{-1}$ is inverse matrix: $\mathbf{D}^{-1}=\frac{\left[D_{r k}\right]^{\mathrm{T}}}{|\mathbf{D}|}, D_{r k}$ is element $d_{r k}$ algebraic complements.

Taking into account (9), we obtain

$$
\hat{m}(n h)=\frac{1}{|\mathbf{D}|} \sum_{j=0}^{2 L} \tilde{m}_{j} f_{j}(n h),
$$

$f_{j}(n h)$ is determined by the following expression:

$$
f_{j}(n h)=D_{j+1,1}+\sum_{j=1}^{L}\left(D_{j+1, r+1} \cos \omega_{r} n h+D_{j+1, r+L+1} \sin \omega_{r} n h\right) .
$$

The mathematical expectations of random quantities $\tilde{m}_{j}$ are equal to

$$
\begin{aligned}
& E \tilde{m}_{0}=m_{0} d_{11}+\sum_{k=1}^{L}\left(m_{k}^{c} d_{1, k+1}+m_{k}^{s} d_{1, L+k+1}\right), \\
& E \tilde{m}_{r}^{c}=m_{0} d_{r+1,1}+\sum_{k=1}^{L}\left(m_{k}^{c} d_{r+1, k+1}+m_{k}^{s} d_{r+1, r+k+1}\right), \\
& E \tilde{m}_{r}^{s}=m_{0} d_{L+r, 1}+\sum_{k=1}^{L}\left(m_{k}^{c} d_{L+r+1, k+1}+m_{k}^{s} d_{L+r+1, L+k+1}\right) .
\end{aligned}
$$

Taking into account the property

$$
\sum_{j=1}^{2 L+1} d_{j r} D_{j k}= \begin{cases}|\mathbf{D}|, & r=1 \\ 0, & r \neq 1\end{cases}
$$

we conclude that $E \hat{m}_{j}=m_{j}$, i.e., the estimators $\hat{m}_{j}$ are unbiased for any sampling interval $h$. Mean estimator (10) is also unbiased.

For variation estimator, we obtain

$$
D[\hat{m}(n h)]=\frac{1}{|\mathbf{D}|^{2}} \sum_{j, l=0}^{2 N_{1}} R_{\tilde{m}_{j} \tilde{m}_{l}} f_{j}(n h) f_{l}(n h),
$$

where

$$
\begin{aligned}
R_{\tilde{m}_{j} \tilde{m}_{r}} & =\frac{1}{K^{2}} \sum_{p, q=0}^{K-1} b[p h,(q-p) h]\left\{\begin{array}{l}
\cos \omega_{j} p h \cos \omega_{r} q h \\
\sin \omega_{j} p h \sin \omega_{r} q h
\end{array}\right\} \\
& =\frac{1}{K^{2}} \sum_{p=0}^{K-1} \sum_{r=-p}^{K-p-1} b(p h, r h)\left\{\begin{array}{l}
\cos \omega_{j} p h \cos \omega_{r}(r+p) h \\
\sin \omega_{j} p h \sin \omega_{j}(r+p) h
\end{array}\right\} .
\end{aligned}
$$


If the covariance function of BPCRP tends to zero as lag $u$ increases, i.e.

$$
\lim _{|u| \rightarrow \infty} b(t, u)=0 \quad \forall t \in \mathbb{R}
$$

then $D[\hat{m}(n h)] \rightarrow 0$ as $K \rightarrow \infty$, i.e. estimator (10) is consistent. For $h \rightarrow 0$ and $\theta=K h$, expression (11) transforms into the formula for the variance of the continuous estimator. The difference between expression (11) and the expression for discrete component estimators of the mean is small when $K$ are big and $h<\pi / \omega_{L}$. Then the error that appears when passing from continuous averaging to discrete averaging is determined by the difference between values of integrals and values of respective integral sums.

\section{LSM-estimators for quadrature model}

Let us concretize above obtained results for quadrature model of BPCRP

$$
\xi(t)=\xi_{c}(t) \cos \Lambda_{11} t+\xi_{s}(t) \sin \Lambda_{11} t
$$

and $E \xi_{c}(t)=m_{c}, E \xi_{s}(t)=m_{s}$ and $\Lambda_{11}=\frac{2 \pi}{T_{1}}+\frac{2 \pi}{T_{2}}$. Functional for estimation of the mean

$$
m(t)=m_{c} \cos \Lambda_{11} t+m_{s} \sin \Lambda_{11} t
$$

is given by

$$
F\left(\hat{m}_{c}, \hat{m}_{s}\right)=\int_{0}^{\theta}\left[\xi(t)-\left(\hat{m}_{c} \cos \Lambda_{11} t+\hat{m}_{s} \sin \Lambda_{11} t\right)\right]^{2} d t .
$$

In discrete case, we have obtained the system of two linear equations with matrix elements

$$
\begin{gathered}
c_{11}=\frac{1}{K} \sum_{n=0}^{K-1} \cos ^{2} \Lambda_{11} n h, \quad s_{11}=\frac{1}{K} \sum_{n=0}^{K-1} \sin ^{2} \Lambda_{11} n h, \quad a_{11}=\frac{1}{K} \sum_{n=0}^{K-1} \cos \Lambda_{11} n h \sin \Lambda_{11} n h, \\
h=\frac{T_{1}}{M+1}, \quad M \in \mathbb{N},
\end{gathered}
$$

and free terms are

$$
\tilde{m}_{c}=\frac{1}{K} \sum_{n=0}^{K-1} \xi(n h) \cos \Lambda_{11} n h, \quad \tilde{m}_{s}=\frac{1}{K} \sum_{n=0}^{K-1} \xi(n h) \sin \Lambda_{11} n h .
$$

The solutions of this system

$$
\hat{m}_{c}=\frac{1}{|\mathbf{D}|}\left(\tilde{m}_{c} s_{11}-\tilde{m}_{s} a_{11}\right), \quad \hat{m}_{s}=\frac{1}{|\mathbf{D}|}\left(\tilde{m}_{s} c_{11}-\tilde{m}_{c} a_{11}\right),
$$

hence

$$
E \tilde{m}_{c}=m_{c} c_{11}+m_{s} a_{11}, \quad E \tilde{m}_{s}=m_{c} a_{11}+m_{s} s_{11},
$$

are unbiased estimators of amplitudes $m_{c}$ and $m_{s}$ for arbitrary value of chosed sampling interval $h$ :

$$
\begin{aligned}
& E \hat{m}_{c}=\frac{1}{|\mathbf{D}|}\left[\left(m_{c} c_{11}+m_{s} a_{11}\right) s_{11}-\left(m_{c} a_{11}+m_{s} s_{11}\right) a_{11}\right]=\frac{1}{|\mathbf{D}|}\left[\begin{array}{c}
m_{c}\left(s_{11} c_{11}-a_{11}^{2}\right) \\
+m_{s}\left(a_{11} s_{11}-s_{11} a_{11}\right)
\end{array}\right]=m_{c}, \\
& E \hat{m}_{s}=\frac{1}{|\mathbf{D}|}\left[\left(m_{c} a_{11}+m_{s} s_{11}\right) c_{11}-\left(m_{c} c_{11}+m_{s} a_{11}\right) a_{11}\right]=\frac{1}{|\mathbf{D}|}\left(c_{11} s_{11}-a_{11}^{2}\right) m_{s}=m_{s} .
\end{aligned}
$$

The variance of the discrete estimator for the mean (13) equals:

Mathematical Modeling and Computing, Vol.6, No. 1, pp. 44-57 (2019) 


$$
D[\hat{m}(n h)]=\frac{1}{|\mathbf{D}|^{2}}\left[D_{\tilde{m}_{c}} f_{1}^{2}(n h)+2 R_{\tilde{m}_{c} \tilde{m}_{s}} f_{1}(n h) f_{2}(n h)+D_{\tilde{m}_{s}} f_{2}^{2}(n h)\right] .
$$

Herewith,

$$
\begin{gathered}
f_{1}(n h)=s_{11} \cos \Lambda_{11} n h-a_{11} \sin \Lambda_{11} n h, \\
f_{2}(n h)=-a_{11} \cos \Lambda_{11} n h+c_{11} \sin \Lambda_{11} n h,
\end{gathered}
$$

and also

$$
\begin{aligned}
D_{\tilde{m}_{c}} & =\frac{1}{K^{2}} \sum_{m, n=0}^{K-1} b[n h,(m-n) h] \cos \Lambda_{11} n h \cos \Lambda_{11} m h \\
& =\frac{1}{2 K^{2}} \sum_{n=0}^{K-1} \sum_{p=-n}^{K-n-1} b(n h, p h)\left[\cos \Lambda_{11} p h+\cos \Lambda_{11}(2 n+p) h\right] \\
D_{\tilde{m}_{s}}= & \frac{1}{K^{2}} \sum_{m, n=0}^{K-1} b[n h,(m-n) h] \sin \Lambda_{11} n h \sin \Lambda_{11} m h \\
= & \frac{1}{2 K^{2}} \sum_{n=0}^{K-1} \sum_{p=-n}^{K-n-1} b(n h, p h)\left[\cos \Lambda_{11} p h-\cos \Lambda_{11}(2 n+p) h\right] \\
R_{\tilde{m}_{c} \tilde{m}_{s}} & =\frac{1}{K^{2}} \sum_{m, n=0}^{K-1} b[n h,(m-n) h] \cos \Lambda_{11} n h \sin \Lambda_{11} m h \\
& =\frac{1}{K^{2}} \sum_{n=0}^{K-1} \sum_{p=-n}^{K-n-1} b(n h, p h) \cos \Lambda_{11} n h \sin \Lambda_{11}(n+p) h .
\end{aligned}
$$

After the summation order changing, we get:

$$
\begin{aligned}
D_{\tilde{m}_{c}}^{(1)}= & \frac{1}{2 K^{2}} \sum_{n=0}^{K-1} \sum_{p=-n}^{K-n-1} b(n h, p h) \cos \Lambda_{11} p h \\
= & \frac{1}{2 K^{2}}\left[\sum_{n=0}^{K-1} b(n h, 0)+2 \sum_{p=0}^{K-1} \sum_{n=0}^{K-p-1} b(n h, p h) \cos \Lambda_{11} p h\right], \\
D_{\tilde{m}_{c}}^{(2)}= & \frac{1}{2 K^{2}} \sum_{n=0}^{K-1} \sum_{p=-n}^{K-n-1} b(n h, p h) \cos \Lambda_{11}(2 n+p) \\
= & \frac{1}{2 K^{2}}\left[\sum_{n=0}^{K-1} b(n h, 0) \cos 2 \Lambda_{11} n h+2 \sum_{p=1}^{K-1} \sum_{n=0}^{K-p-1} b(n h, p h) \cos \Lambda_{11}(2 n+p) h\right] \\
R_{\tilde{m}_{c} \tilde{m}_{s}}= & \frac{1}{2 K^{2}}\left[\sum_{n=0}^{K-1} b(n h, 0) \sin 2 \Lambda_{11} n h\right. \\
& \left.+2 \sum_{p=1}^{K-1} \sum_{n=0}^{K-p-1} b(n h, p h)\left[\cos \Lambda_{11} n h \sin \Lambda_{11}(n+p) h+\cos \Lambda_{11}(n+p) h \sin \Lambda_{11} n h\right]\right] .
\end{aligned}
$$

Mathematical Modeling and Computing, Vol.6, No. 1, pp. 44-57 (2019) 
Let us substitute the formula for covariance function of the random process (12)

$$
b(n h, p h)=B_{00}(p h)+\sum_{r= \pm 2} B_{r r}(p h) e^{i \Lambda_{r r} p h}
$$

into these relationships. Then,

$$
\begin{aligned}
D_{\tilde{m}_{c}}^{(1)}= & \frac{1}{2 K}\left[B_{00}(0)+\sum_{r= \pm 2}\left[B_{r r}(0) f_{r r}(0, K-1)+\sum_{p=1}^{K-1} B_{r r}(p h) f_{r r}(0, K-p-1) \cos \Lambda_{r r} p h\right]\right], \\
D_{\tilde{m}_{c}}^{(2)}= & \frac{1}{4 K}\left[\sum _ { r = 0 , \pm 2 } \left[B_{r r}(0)\left[f_{r+2, r+2}(0, K-1)+f_{r-2, r-2}(0, K-1)\right]\right.\right. \\
& \left.\left.+2 \sum_{p=1}^{K-1} B_{r r}(p h)\left[e^{i \Lambda_{r r} p h} f_{r+2, r+2}(0, K-p-1)+e^{-i \Lambda_{r r} p h} f_{r-2, r-2}(0, K-p-1)\right]\right]\right],
\end{aligned}
$$

where

$$
f_{r r}(0, K-1)=\frac{1}{K} \sum_{n=0}^{K-1} e^{i \Lambda_{r r} n h} .
$$

Components of these expressions depend on the functions $f_{r r}(0, K-1)$ and $f_{r r}(0, K-p-1), r \neq 0$, are the quantities of the highest order of smallness as compared with the zero components. Neglecting them, we obtain the following formulae for quantities (15) and (16):

$$
\begin{aligned}
& D_{\tilde{m}_{c}}=\frac{1}{2 K}\left[B_{00}(0)+\frac{1}{2} B_{22}^{c}(0)+\sum_{p=1}^{K-1}\left(1-\frac{p}{K}\right)\left[2 B_{00}(p h) \cos \Lambda_{11} p h\right.\right. \\
& \left.+B_{22}^{c}(p h) \cos \Lambda_{11} p h-B_{22}^{s}(p h) \sin \Lambda_{11} p h\right], \\
& D_{\tilde{m}_{s}}=\frac{1}{2 K}\left[B_{00}(0)-\frac{1}{2} B_{22}^{c}(0)+\sum_{p=1}^{K-1}\left(1-\frac{p}{K}\right)\left[2 B_{00}(p h) \cos \Lambda_{11} p h\right.\right. \\
& \left.\left.-B_{22}^{c}(p h) \cos \Lambda_{11} p h+B_{22}^{s}(p h) \sin \Lambda_{11} p h\right]\right] .
\end{aligned}
$$

After analogic transformations of the expression (17) at a first approximation we get:

$$
R_{\tilde{m}_{c} \tilde{m}_{s}}=\frac{1}{2 K}\left[B_{22}^{s}(0)+2 \sum_{p=1}^{K-1}\left(1-\frac{p}{K}\right)\left[B_{22}^{s}(p h) \cos \lambda_{11} p h+B_{22}^{c}(p h) \sin \lambda_{11} p h\right]\right] .
$$

Let us represent covariance components through the auto- and crosscovariance functions of quadrature components:

$$
\begin{aligned}
& B_{00}(p h)=\frac{1}{2}\left[R_{c}(p h)+R_{s}(p h)\right] \cos \Lambda_{11} p h+R_{c s}^{-}(p h) \sin \Lambda_{11} p h, \\
& B_{22}^{c}(p h)=\frac{1}{2}\left[R_{c}(p h)-R_{s}(p h)\right] \cos \Lambda_{11} p h+R_{c s}^{+}(p h) \sin \Lambda_{11} p h, \\
& B_{22}^{s}(p h)=\frac{1}{2}\left[R_{s}(p h)-R_{c}(p h)\right] \sin \Lambda_{11} p h+R_{c s}^{+}(p h) \cos \Lambda_{11} p h .
\end{aligned}
$$

Mathematical Modeling and Computing, Vol.6, No. 1, pp. 44-57 (2019) 
Here $R_{c}(p h)$ and $R_{s}(p h)$ are autocovariance functions of quadrature components: $R_{c}(p h)=$ $E \stackrel{\circ}{\xi}_{c}(n h) \stackrel{\circ}{\xi}_{s}((n+p) h), R_{s}(p h)=E \stackrel{\circ}{\xi_{s}}(n h) \stackrel{\circ}{\xi_{s}}((n+p) h), \stackrel{\circ}{\xi_{c}}(n h)=\xi_{c}(n h)-m_{c}, \stackrel{\circ}{\xi}_{s}(n h)=\xi_{s}(n h)-m_{s}$, $m_{c}=E \xi_{c}(n h), m_{s}=E \xi_{s}(n h) ; R_{c s}^{+}(p h)$ and $R_{c s}^{-}(p h)$ are the odd and even parts of crosscovariance function: $R_{c s}(p h)=E \stackrel{\circ}{\xi_{c}}(n h) \stackrel{\circ}{\xi_{s}}((n+p) h)$. If we set $R_{c s}^{-}(p h)=0$, after transformations we obtain

$$
\begin{aligned}
D_{\tilde{m}_{c}} & =\frac{1}{2 K}\left[\frac{1}{4}\left(3 R_{c}(0)+R_{s}(0)\right)+\sum_{p=1}^{K-1}\left(1-\frac{p}{K}\right)\left[R_{c}(p h)+\frac{1}{2}\left[R_{c}(p h)+R_{s}(p h)\right] \cos \Lambda_{22} p h\right]\right], \\
D_{\tilde{m}_{s}} & =\frac{1}{2 K}\left[\frac{1}{4}\left(R_{c}(0)+3 R_{s}(0)\right)+\sum_{p=1}^{K-1}\left(1-\frac{p}{K}\right)\left[R_{c}(p h)+R_{s}(p h)\right] \cos \Lambda_{22} p h\right], \\
R_{\tilde{m}_{c} \tilde{m}_{s}} & =\frac{1}{2 K}\left[R_{c s}^{+}(0)+2 \sum_{p=1}^{K-1}\left(1-\frac{p}{K}\right) R_{c s}^{+}(p h)\right] .
\end{aligned}
$$

Accepting

$$
R_{c}(p h)=D_{1} e^{-\alpha_{1}|p| h}, \quad R_{s}(p h)=D_{2} e^{-\alpha_{2}|p| h}, \quad R_{c s}^{+}(p h)=D_{3} e^{-\alpha_{3}|p| h}
$$

and introducing the functions

$$
\begin{aligned}
& s_{0}\left(\alpha_{i}, K\right)=\frac{1}{2 K} \sum_{p=1}^{K-1}\left(1-\frac{p}{K}\right) e^{-\alpha_{i} p h} \\
& s_{2}\left(\alpha_{i}, K\right)=\frac{1}{2 K} \sum_{p=1}^{K-1}\left(1-\frac{p}{K}\right) e^{-\alpha_{i} p h} \cos \Lambda_{22} p h
\end{aligned}
$$

we have

$$
\begin{aligned}
D_{\tilde{m}_{c}} & =\frac{1}{8 K}\left(3 D_{1}+D_{2}\right)+D_{1} s_{0}\left(\alpha_{1}, K\right)+\frac{1}{2}\left[D_{1} s_{2}\left(\alpha_{1}, K\right)+D_{2} s_{2}\left(\alpha_{2}, K\right)\right], \\
D_{\tilde{m}_{s}} & =\frac{1}{8 K}\left(D_{1}+3 D_{2}\right)+D_{2} s_{0}\left(\alpha_{2}, K\right)+\frac{1}{2}\left[D_{1} s_{2}\left(\alpha_{1}, K\right)+D_{2} s_{2}\left(\alpha_{2}, K\right)\right], \\
R_{\tilde{m}_{c} \tilde{m}_{s}} & =\frac{D_{s}}{2 K}+2 D_{3} s_{0}\left(\alpha_{3}, K\right) .
\end{aligned}
$$

Taking into account

$$
\begin{aligned}
f_{1}^{2}(n h) & =\frac{1}{2}\left(s_{11}^{2}+a_{11}^{2}\right)-s_{11} a_{11} \sin \Lambda_{22} n h+\frac{1}{2}\left(s_{11}^{2}-a_{11}^{2}\right) \cos \Lambda_{22} n h, \\
f_{2}^{2}(n h) & =\frac{1}{2}\left(c_{11}^{2}+a_{11}^{2}\right)-c_{11} a_{11} \sin \Lambda_{22} n h+\frac{1}{2}\left(a_{21}^{2}-a_{22}^{2}\right) \cos \Lambda_{22} n h, \\
f_{1}(n h) f_{2}(n h) & =-\frac{a_{11}}{2}\left(s_{11}+c_{11}\right)+\frac{a_{11}}{2}\left(c_{11}-s_{11}\right) \cos \Lambda_{22} n h+\frac{1}{2}\left(c_{11} s_{11}+a_{11}^{2}\right) \sin \Lambda_{22} n h,
\end{aligned}
$$

we can rewrite variance (14) in the following form:

$$
\begin{aligned}
D[\hat{m}(n h)] & =D_{0}[\hat{m}(n h)]+D_{c}[\hat{m}(n h)] \cos \Lambda_{22} n h+D_{s}[\hat{m}(n h)] \sin \Lambda_{22} n h \\
& =D_{0}[\hat{m}(n h)]+D_{2}[\hat{m}(n h)] \cos \left(\Lambda_{22} n h-\varphi_{22}(n h)\right),
\end{aligned}
$$

where

$$
D_{0}[\hat{m}(n h)]=\frac{1}{|\mathbf{D}|^{2}}\left[D_{\tilde{m}_{c}}\left(s_{11}^{2}+a_{11}^{2}\right)+D_{\tilde{m}_{s}}\left(c_{11}^{2}+a_{11}^{2}\right)-2 R_{\tilde{m}_{c} \tilde{m}_{s}}\left(s_{11} a_{11}+c_{11} a_{11}\right)\right]
$$




$$
\begin{aligned}
& D_{c}[\hat{m}(n h)]=\frac{1}{2|\mathbf{D}|^{2}}\left[D_{\tilde{m}_{c}}\left(s_{11}^{2}-a_{11}^{2}\right)+D_{\tilde{m}_{s}}\left(a_{11}^{2}-c_{11}^{2}\right)-2 R_{\tilde{m}_{c} \tilde{m}_{s}}\left(s_{11} a_{11}-c_{11} a_{11}\right)\right], \\
& D_{s}[\hat{m}(n h)]=\frac{1}{|\mathbf{D}|^{2}}\left[2 R_{\tilde{m}_{c} \tilde{m}_{s}}\left(s_{11} c_{11}+a_{11}^{2}\right)-D_{\tilde{m}_{c}} s_{11} a_{11}-D_{\tilde{m}_{s}} c_{11} a_{11}\right], \\
& D_{2}[\hat{m}(n h)]=\left[\left[D_{c}[\hat{m}(n h)]\right]^{2}+\left[D_{s}[\hat{m}(n h)]\right]^{2}\right]^{\frac{1}{2}}, \quad \varphi_{22}(n h)=\arctan \frac{D_{s}[\hat{m}(n h)]}{D_{c}[\hat{m}(n h)]},
\end{aligned}
$$

and

$$
c_{11}=\frac{1}{2}\left(1+\frac{\sin \Lambda_{22} \theta}{\Lambda_{22} \theta}\right), \quad s_{11}=\frac{1}{2}\left(1-\frac{\sin \Lambda_{22} \theta}{\Lambda_{22} \theta}\right), \quad a_{11}=\frac{1}{2}\left(1-\frac{\cos \Lambda_{22} \theta}{2 \Lambda_{22} \theta}\right) .
$$

The variance components (18) were calculated for chosen values of parameters $D_{i}, \alpha_{i}$, different ratios $T_{1}$ and $T_{2}$, and also length of realization and sampling interval used the obtained formulae. The results of calculation are given in Table 3 .

Table 3. Values of quantities $D_{0}[\hat{m}(n h)]$ and $D_{2}[\hat{m}(n h)]$ for different damping decrements when $\theta=100 T_{1}$, $T_{2}=T_{1}, T_{1}=10 \mathrm{~s}$, and $h=\frac{T_{1}}{M+1}$.

\begin{tabular}{|c|c|c|c|c|c|c|}
\hline \multirow{2}{*}{$M$} & \multicolumn{2}{|c|}{$\alpha_{1}=\alpha_{2}=0.02 ; \alpha_{3}=0.01$} & \multicolumn{2}{|c|}{$\alpha_{1}=\alpha_{2}=0.6 ; \alpha_{3}=0.3$} & \multicolumn{2}{c|}{$\alpha_{1}=\alpha_{2}=1 ; \alpha_{3}=0.5$} \\
\cline { 2 - 7 } & $D_{0}[\hat{m}(n h)]$ & $D_{2}[\hat{m}(n h)]$ & $D_{0}[\hat{m}(n h)]$ & $D_{2}[\hat{m}(n h)]$ & $D_{0}[\hat{m}(n h)]$ & $D_{2}[\hat{m}(n h)]$ \\
\hline 5 & $0.7603 \cdot 10^{-1}$ & $0.5772 \cdot 10^{-1}$ & $0.3498 \cdot 10^{-2}$ & $0.2635 \cdot 10^{-2}$ & $0.2863 \cdot 10^{-2}$ & $0.1833 \cdot 10^{-2}$ \\
\hline 10 & $0.7601 \cdot 10^{-1}$ & $0.5750 \cdot 10^{-1}$ & $0.3050 \cdot 10^{-2}$ & $0.2378 \cdot 10^{-2}$ & $0.2185 \cdot 10^{-2}$ & $0.1551 \cdot 10^{-2}$ \\
\hline 15 & $0.7601 \cdot 10^{-1}$ & $0.5742 \cdot 10^{-1}$ & $0.2973 \cdot 10^{-2}$ & $0.2288 \cdot 10^{-2}$ & $0.2057 \cdot 10^{-2}$ & $0.1455 \cdot 10^{-2}$ \\
\hline 20 & $0.7601 \cdot 10^{-1}$ & $0.5738 \cdot 10^{-1}$ & $0.2945 \cdot 10^{-2}$ & $0.2242 \cdot 10^{-2}$ & $0.2012 \cdot 10^{-2}$ & $0.1406 \cdot 10^{-2}$ \\
\hline 25 & $0.7601 \cdot 10^{-1}$ & $0.5735 \cdot 10^{-1}$ & $0.2932 \cdot 10^{-2}$ & $0.2214 \cdot 10^{-2}$ & $0.1990 \cdot 10^{-2}$ & $0.1377 \cdot 10^{-2}$ \\
\hline 30 & $0.7601 \cdot 10^{-1}$ & $0.5734 \cdot 10^{-1}$ & $0.2925 \cdot 10^{-2}$ & $0.2196 \cdot 10^{-2}$ & $0.1978 \cdot 10^{-2}$ & $0.1358 \cdot 10^{-2}$ \\
\hline 40 & $0.7601 \cdot 10^{-1}$ & $0.5731 \cdot 10^{-1}$ & $0.2917 \cdot 10^{-2}$ & $0.2172 \cdot 10^{-2}$ & $0.1966 \cdot 10^{-2}$ & $0.1334 \cdot 10^{-2}$ \\
\hline 50 & $0.7601 \cdot 10^{-1}$ & $0.5731 \cdot 10^{-1}$ & $0.2914 \cdot 10^{-2}$ & $0.2158 \cdot 10^{-2}$ & $0.1960 \cdot 10^{-2}$ & $0.1320 \cdot 10^{-2}$ \\
\hline 100 & $0.7601 \cdot 10^{-1}$ & $0.5728 \cdot 10^{-1}$ & $0.2909 \cdot 10^{-2}$ & $0.2129 \cdot 10^{-2}$ & $0.1953 \cdot 10^{-2}$ & $0.1291 \cdot 10^{-2}$ \\
\hline 150 & $0.7601 \cdot 10^{-1}$ & $0.5725 \cdot 10^{-1}$ & $0.2909 \cdot 10^{-2}$ & $0.2120 \cdot 10^{-2}$ & $0.1951 \cdot 10^{-2}$ & $0.1282 \cdot 10^{-2}$ \\
\hline 200 & $0.7601 \cdot 10^{-1}$ & $0.5724 \cdot 10^{-1}$ & $0.2908 \cdot 10^{-2}$ & $0.2116 \cdot 10^{-2}$ & $0.1951 \cdot 10^{-2}$ & $0.1277 \cdot 10^{-2}$ \\
\hline $\begin{array}{c}\text { Continuous } \\
\text { estimator }\end{array}$ & $0.7601 \cdot 10^{-1}$ & $0.5724 \cdot 10^{-1}$ & $0.2907 \cdot 10^{-2}$ & $0.2101 \cdot 10^{-2}$ & $0.1950 \cdot 10^{-2}$ & $0.1263 \cdot 10^{-2}$ \\
\hline
\end{tabular}

The variance of the continuous estimators [9] were calculated also. In this case we have:

$$
\begin{aligned}
D_{\tilde{m}_{c}} & =D_{1} r_{0}\left(\alpha_{1}, \theta\right)+\frac{1}{2}\left[D_{1} r_{2}\left(\alpha_{1}, \theta\right)+D_{2} r_{2}\left(\alpha_{2}, \theta\right)\right], \\
D_{\tilde{m}_{s}} & =D_{2} r_{0}\left(\alpha_{2}, \theta\right)+\frac{1}{2}\left[D_{1} r_{2}\left(\alpha_{1}, \theta\right)+D_{2} r_{2}\left(\alpha_{2}, \theta\right)\right], \\
R_{\tilde{m}_{c} \tilde{m}_{s}} & =D_{3} r_{0}\left(\alpha_{3}, \theta\right),
\end{aligned}
$$

where

$$
\begin{aligned}
& r_{0}\left(\alpha_{i}, \theta\right)=\frac{1}{2 \theta} \int_{0}^{\theta}\left(1-\frac{u}{\theta}\right) e^{-\alpha_{i} u} d u, \\
& r_{2}\left(\alpha_{i}, \theta\right)=\frac{1}{2 \theta} \int_{0}^{\theta}\left(1-\frac{u}{\theta}\right) e^{-\alpha_{i} u} \cos \Lambda_{22} u d u .
\end{aligned}
$$

Mathematical Modeling and Computing, Vol.6, No. 1, pp. 44-57 (2019) 
As can be seen from the Table 3, the difference between the values of the variance for discrete estimator and the values of the variance of continuous estimator are small if the damping coefficients are small. The chosen number of a sampling points on the period $T_{1}$ is greater than 10, i.e., two times greater than the number provided absence of aliasing effect of the second kind [10,11]. If damping coefficients increase the value of the variances in discrete and continuous cases and also difference between them increase too. For example, we have $D_{0}[\hat{m}(n h)]=1.12 D[\hat{m}(t)]$ and $D_{2}[\hat{m}(n h)]=$ $1.22 D[\hat{m}(t)]$ if $M=10$ and $\alpha_{1}=\alpha_{2}=1 ; \alpha_{3}=0.5$. The value of variances decrease slowly if sampling interval decreases. The inequality $D_{2}[\hat{m}(n h)]<1.05 D_{2}[\hat{m}(t)]$ is satisfied only if $M \geqslant 50$. The calculations carried out for $T_{2}=1.5 T_{1}$ and $T_{2}=1.1 T_{1}$ show that the properties of the variance part are similar.
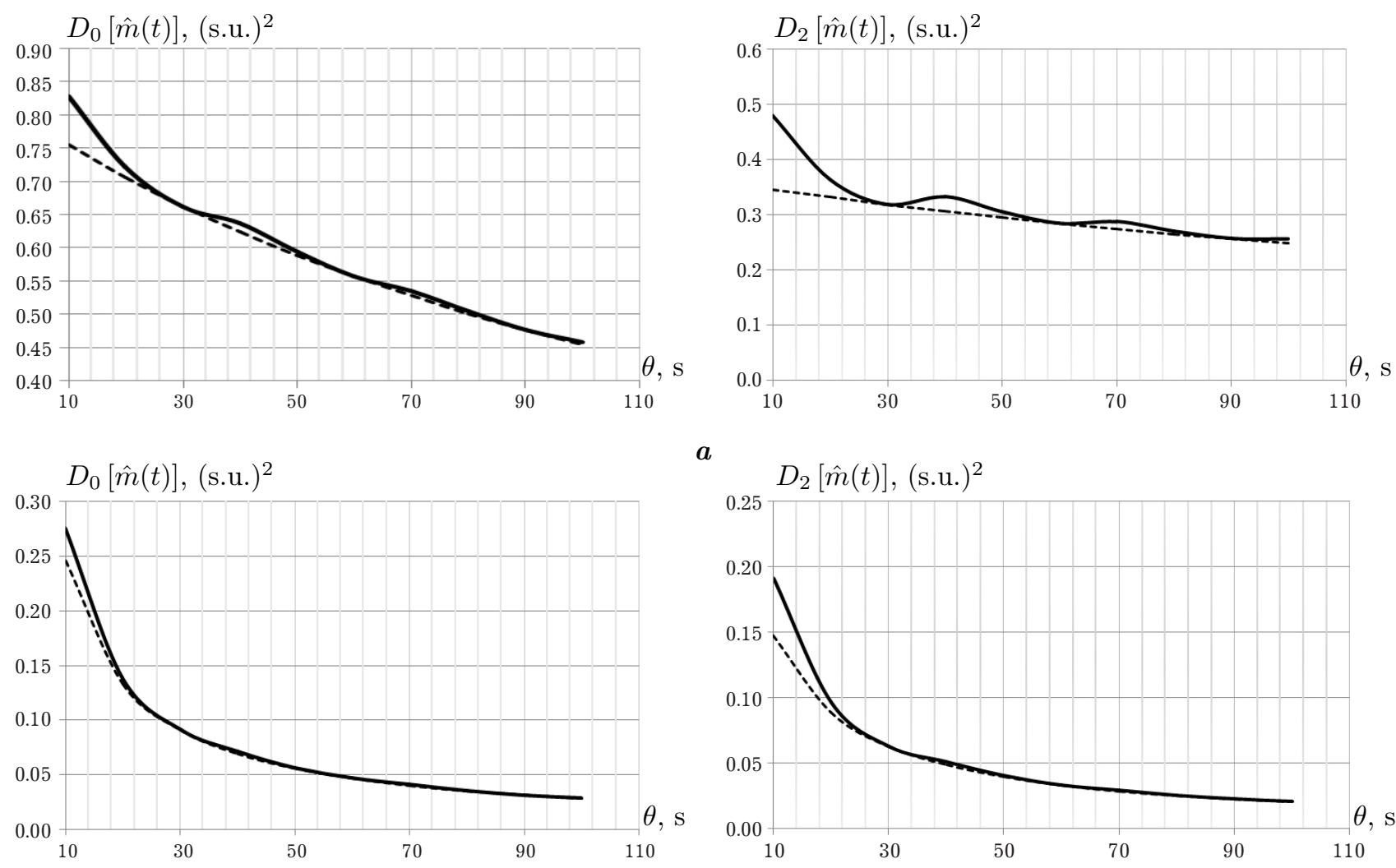

$\boldsymbol{a}$
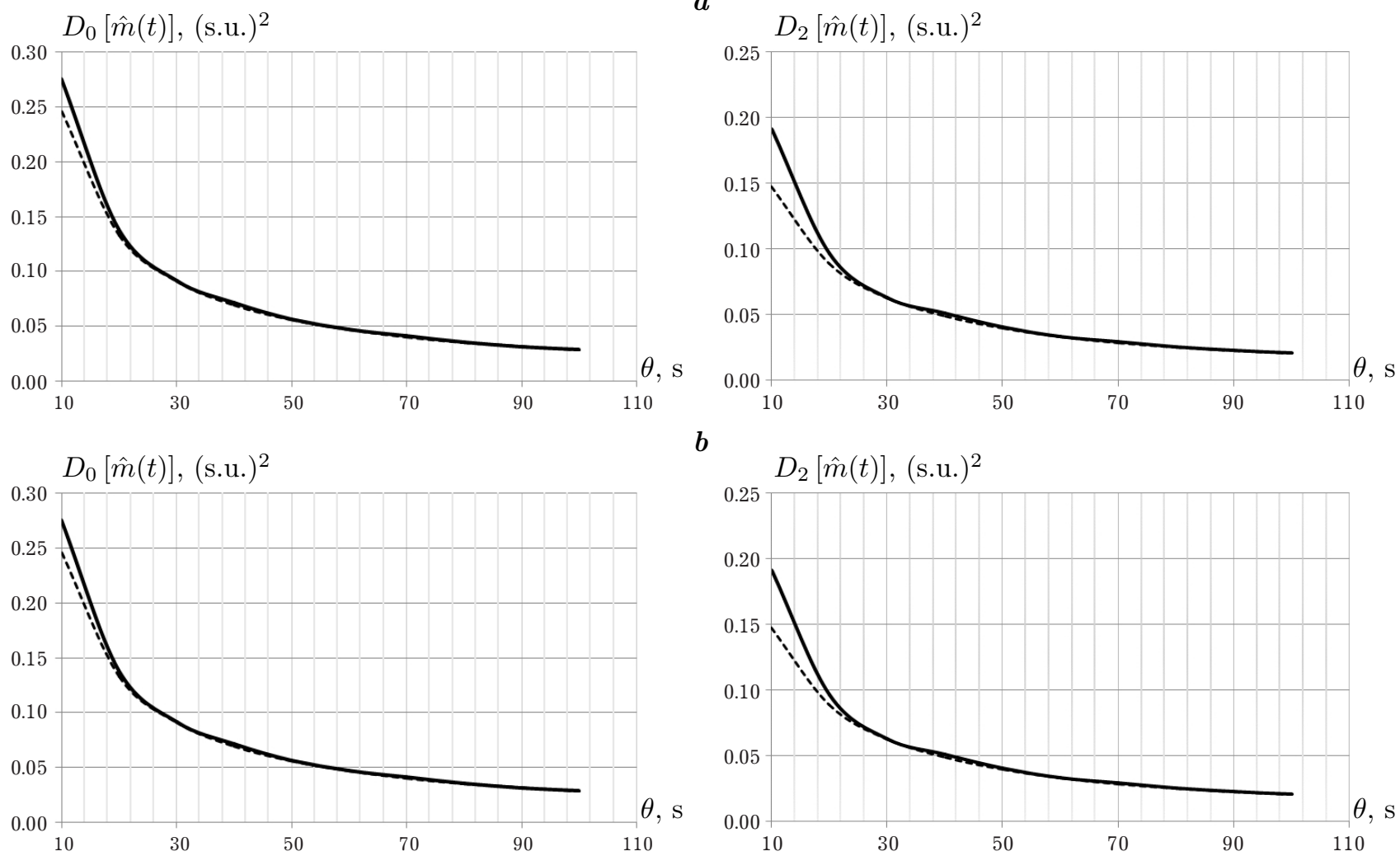

$b$

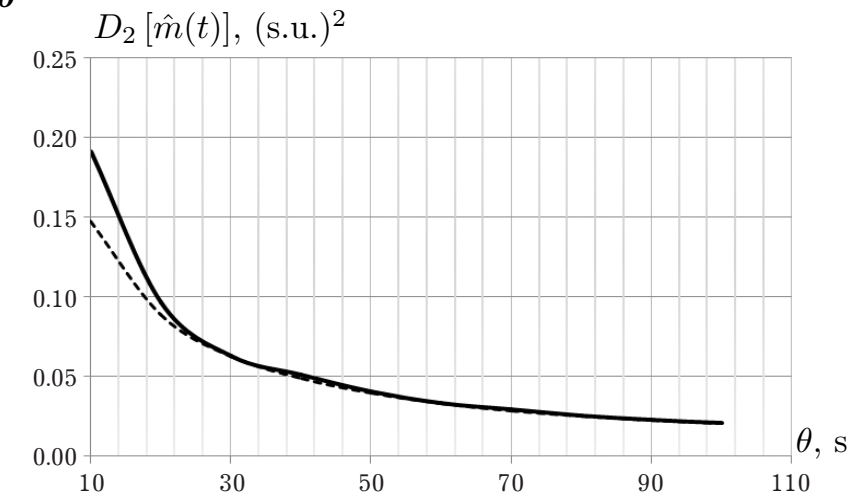

$c$

Fig. 1. Dependencies of variance parts of component estimator (---) and LSM-estimator (-) on the realization length: $(a) \alpha_{1}=\alpha_{2}=0.02 ; \alpha_{3}=0.01 ;(b) \alpha_{1}=\alpha_{2}=0.6 ; \alpha_{3}=0.3 ;(c) \alpha_{1}=\alpha_{2}=1 ; \alpha_{3}=0.5$. 
Let us compare the obtained numerical results with the results when component method is used. For continuous estimator

$$
\hat{m}(t)=\left[\frac{2}{\theta} \int_{0}^{\theta} \xi(s) \cos \Lambda_{11} s d s\right] \cos \Lambda_{11} t+\left[\frac{2}{\theta} \int_{0}^{\theta} \xi(s) \sin \Lambda_{11} s d s\right] \sin \Lambda_{11} t
$$

we have

$$
D_{0}[\hat{m}(t)]=D\left[\hat{m}_{c}\right]+D\left[\hat{m}_{s}\right], \quad D_{2}[\hat{m}(t)]=\left[\left(R_{\hat{m}_{c} \hat{m}_{s}}\right)^{2}+\left[D\left[\hat{m}_{c}\right]-D\left[\hat{m}_{s}\right]\right]^{2}\right]^{\frac{1}{2}},
$$

where

$$
\begin{gathered}
D\left[\hat{m}_{c}\right]=2 D_{1} r_{0}\left(\alpha_{1}, \theta\right)+D_{1} r_{2}\left(\alpha_{1}, \theta\right)+D_{2} r_{2}\left(\alpha_{2}, \theta\right), \\
D\left[\hat{m}_{s}\right]=2 D_{2} r_{0}\left(\alpha_{2}, \theta\right)+D_{1} r_{2}\left(\alpha_{1}, \theta\right)+D_{2} r_{2}\left(\alpha_{2}, \theta\right), \\
R_{\tilde{m}_{c} \tilde{m}_{s}}=4 D_{3} r_{0}\left(\alpha_{3}, \theta\right) .
\end{gathered}
$$

The results of calculations are shown graphically in the form of dependencies of variance parts on the realization length $\theta$ for different damping decrements when $T_{2}=1.5 T_{1}, T_{1}=10 \mathrm{~s}$. As it is seen, the values of variance of LSM-estimator rapidly converge to the values of component estimator and when $\theta>10 T_{1}$, these values are almost the same. For small damping coefficients, this convergence has an oscillatory form, and for great decrements it has a monotonous form. Similar results were obtained when we calculated variances of coefficients for another relationships between periods $T_{1}$ and $T_{2}$.

\section{Conclusion}

Therefore, using the LSM-estimation of a mean function gives us an opportunity to avoid systematical error caused by a leakage effect. Meanwhile, the values of root-mean-squar (rms) errors of component estimators and LSM-estimators differ insignificantly even in cases when realization length is small. The concrete values of rsm errors in dependence on realization length and sampling interval can be calculated based on the formulae obtained in the present paper. Realization length $\theta$ (sample number $K$ ) and the value of sampling interval that provides necessary rsm error of processing depend significantly on the values of damping coefficients of correlations. If coefficients decrements are great, the sampling interval that provides this error can be dozens times greater than the interval which provides the absence of aliasing effects of the first and second kinds.

[1] Javors'kyj I., Yuzefovych R., Matsko I., Kravets I. The Stochastic Recurrence Structure of Geophysical Phenomena. 55-88 (2015). In: Chaari F., Leskow J., Napolitano A., Zimroz R., Wylomanska A., Dudek A. (eds) Cyclostationarity: Theory and Methods - II. CSTA 2014. Applied Condition Monitoring, vol 3. Springer, Cham.

[2] Denysenko N. A., Hoffman I., Inshekov E. N. Simplified stochastic model of the electric load in electricity system. Russian Electromechanics. 8, 104-108 (1987).

[3] Napolitano A. Generalizations of Cyclostationary Signal Processing: Spectral Analysis and Applications. John Wiley \& Sons, Ltd. IEEE Press (2012).

[4] Javorskyj I., Kravets I., Matsko I., Yuzefovych R. Periodically correlated random processes: Application in early diagnostics of mechanical systems. Mechanical Systems and Signal Processing. 83, 406-438 (2017).

[5] Antoni J. Cyclostationarity by examples. Mechanical Systems and Signal Processing. 23, 987-1036 (2009).

[6] Javorskyj I. On period estimate of periodically correlated random processes. Otbor i Peredacha Informatsiyi. 73, 12-21 (1986), (in Russian).

[7] Dragan Y., Rozhkov V., Javorskyj I. The Methods of Probabilistic Analysis of Oceanological Rhythms. Leningrad, Gidrometeoizdat (1987), (in Russian). 
[8] Javorskyj I. Mathematical models and analysis of stochastic oscillations. Lviv, Physico-mechanical institute of NAS of Ukraine (2013), (in Ukrainian).

[9] Javorskyj I., Yuzefovych R., Dzeryn O. LSM-harmonic analysis of bi-periodic nonstationary vibration signals. Information extraction and processing. 45 (121), 14-25 (2017), (in Ukrainian).

[10] Javorskyj I., Matsko I., Yuzefovych R., Zakrzewski Z. Discrete estimators of characteristics for periodically correlated time series. Digital Signal Processing. 53, 25-40 (2016).

[11] Javorskyj I., Yuzefovych R., Kravets I., Matsko I. Properties of characteristics estimators of periodically correlated random processes in preliminary determination of the period of correlation. Radioelectronics and Communication Systems. 55 (8), 335-348 (2012).

\title{
Аналіз дискретної МНК-оцінки математичного сподіванння біперіодично нестаціонарних випадкових сигналів
}

\author{
Яворський I. ${ }^{1,2}$, Дзерин O. ${ }^{1}$, Юзефович P. ${ }^{1,3}$ \\ ${ }^{1}$ Фізико-механічний інститут ім. Г. В. Карпенка НАН Украӥни, \\ лабораторія вібродіагностики, \\ вул. Наукова, 5, Лъвів, 79060, Україна \\ ${ }^{2}$ Технологічно-природничий університет, \\ Інститут телекомунікації та інформатики, \\ алея проф. С. Каліського, 7, Бидгощ, 85796, Польща \\ ${ }^{3}$ Націоналъний університет "Львівсъка політехніка", \\ кафедра прикладної математики, \\ вул. С. Бандери, 12, Лъвів, 79013, Україна
}

Проаналізовано дискретні оцінки детермінованої складової біперіодично нестаціонарних випадкових сигналів, отриманих за допомогою методів найменших квадратів (MHK). Показано, що МНК-оцінювання дає можливість уникнути ефектів просочування. Визначено умови слушності дискретних оцінок. Проаналізовано формули для дисперсії оцінок, які описують її залежність від довжини реалізації, інтервалу дискретизації та кореляційних компонентів сигналу.

Ключові слова: біперіодично корелвовані випадкові прочеси, метод найменших квадратів, дискретні оцінки параметрів детермінованої складової, незміщеність очінки, слушність.

2000 MSC: 60G35, 94A12

УдК: $621.391: 519.72$ 